\title{
A Numerical Iterative Method for Solving Systems of First-Order Periodic Boundary Value Problems
}

\author{
Mohammed AL-Smadi, ${ }^{1}$ Omar Abu Arqub, ${ }^{2}$ and Ahmad El-Ajou ${ }^{2}$ \\ ${ }^{1}$ Applied Science Department, Ajloun College, Al-Balqa Applied University, Ajloun 26816, Jordan \\ ${ }^{2}$ Department of Mathematics, Al-Balqa Applied University, Salt 19117, Jordan
}

Correspondence should be addressed to Omar Abu Arqub; o.abuarqub@bau.edu.jo

Received 12 September 2013; Accepted 11 February 2014; Published 25 March 2014

Academic Editor: Hak-Keung Lam

Copyright (C) 2014 Mohammed AL-Smadi et al. This is an open access article distributed under the Creative Commons Attribution License, which permits unrestricted use, distribution, and reproduction in any medium, provided the original work is properly cited.

\begin{abstract}
The objective of this paper is to present a numerical iterative method for solving systems of first-order ordinary differential equations subject to periodic boundary conditions. This iterative technique is based on the use of the reproducing kernel Hilbert space method in which every function satisfies the periodic boundary conditions. The present method is accurate, needs less effort to achieve the results, and is especially developed for nonlinear case. Furthermore, the present method enables us to approximate the solutions and their derivatives at every point of the range of integration. Indeed, three numerical examples are provided to illustrate the effectiveness of the present method. Results obtained show that the numerical scheme is very effective and convenient for solving systems of first-order ordinary differential equations with periodic boundary conditions.
\end{abstract}

\section{Introduction}

Systems of ordinary differential equations with periodic boundary value conditions, the so-called periodic boundary value problems (BVPs), are well known for their applications in sciences and engineering [1-5]. In this paper, we focus on finding approximate solutions to systems of first-order periodic BVPs, which are a combination of systems of firstorder ordinary differential equations and periodic boundary conditions. In fact, accurate and fast numerical solutions of systems of first-order periodic BVPs are of great importance due to their wide applications in scientific and engineering research.

Numerical methods are becoming more and more important in mathematical and engineering applications, simply not only because of the difficulties encountered in finding exact analytical solutions but also because of the ease with which numerical techniques can be used in conjunction with modern high-speed digital computers. A numerical procedure for solving systems of first-order periodic BVPs based on the use of reproducing kernel Hilbert space (RKHS) method is discussed in this work.

Among a substantial number of works dealing with systems of first-order periodic BVPs, we mention [6-10]. The existence of solutions to systems of first-order periodic BVPs has been discussed as described in [6]. In [7], the authors have discussed some existence and uniqueness results of periodic solutions for first-order periodic differential systems. Also, in [8] the authors have provided the existence, multiplicity, and nonexistence of positive periodic solutions for systems of first-order periodic BVPs. Furthermore, the existence of periodic solutions for the coupled first-order differential systems of Hamiltonian type is carried out in [9]. Recently, the existence of positive solutions for systems of first-order periodic BVPs is proposed in [10]. For more results on the solvability analysis of solutions for systems of firstorder periodic BVPs, we refer the reader to [11-15], and for numerical solvability of different categories of BVPs, one can consult [16-19]. 
Investigation about systems of first-order periodic BVPs numerically is scarce. In this paper, we utilize a methodical way to solve these types of differential systems. In fact, we provide criteria for finding the approximate and exact solutions to the following system:

$$
\begin{gathered}
u_{1}^{\prime}(x)=F_{1}\left(x, u_{1}(x), u_{2}(x), \ldots, u_{n}(x)\right), \\
u_{2}^{\prime}(x)=F_{2}\left(x, u_{1}(x), u_{2}(x), \ldots, u_{n}(x)\right), \\
\vdots \\
u_{n}^{\prime}(x)=F_{n}\left(x, u_{1}(x), u_{2}(x), \ldots, u_{n}(x)\right),
\end{gathered}
$$

subject to the periodic boundary conditions

$$
\begin{gathered}
u_{1}(0)=u_{1}(1), \\
u_{2}(0)=u_{2}(1), \\
\vdots \\
u_{n}(0)=u_{n}(1),
\end{gathered}
$$

where $x \in[0,1], u_{s} \in W_{2}^{2}[0,1]$ are unknown functions to be determined, $F_{s}\left(x, v_{1}, v_{1}, \ldots, v_{n}\right)$ are continuous terms in $W_{2}^{1}[0,1]$ as $v_{s}=v_{s}(x) \in W_{2}^{2}[0,1], 0 \leq x \leq 1,-\infty<v_{s}<$ $\infty$ in which $s=1,2, \ldots, n$, and $W_{2}^{1}[0,1], W_{2}^{2}[0,1]$ are two reproducing kernel spaces. Here, we assume that (1) subject to the periodic boundary conditions (2) has a unique solution on $[0,1]$.

Reproducing kernel theory has important applications in numerical analysis, differential equations, integral equations, probability and statistics, and so forth [20-22]. In the last years, extensive work has been done using RKHS method which provides numerical approximations for linear and nonlinear equations. This method has been implemented in several operator, differential, integral, and integrodifferential equations side by side with their theories. The reader is kindly requested to go through [23-35] in order to know more details about RKHS method, including its history, its modification for use, its applications, and its characteristics.

The rest of the paper is organized as follows. In the next section, two reproducing kernel spaces are described in order to formulate the reproducing kernel functions. In Section 3, some essential results are introduced and a method for the existence of solutions for (1) and (2) is described. In Section 4, we give an iterative method to solve (1) and (2) numerically. Numerical examples are presented in Section 5. Section 6 ends this paper with brief conclusions.

\section{Construct of Reproducing Kernel Functions}

In this section, two reproducing kernels needed are constructed in order to solve (1) and (2) using RKHS method. Before the construction, we utilize the reproducing kernel concept. Throughout this paper, $\mathbb{C}$ is the set of complex numbers, $L^{2}[a, b]=\left\{u \mid \int_{a}^{b} u^{2}(x) d x<\infty\right\}$, and $l^{2}=\{A \mid$ $\left.\sum_{i=1}^{\infty}\left(A_{i}\right)^{2}<\infty\right\}$.

Definition 1 (see [23]). Let $E$ be a nonempty abstract set. A function $R: E \times E \rightarrow \mathbb{C}$ is a reproducing kernel of the Hilbert space $H$ if
(1) for each $x \in E, R(\cdot, x) \in H$

(2) for each $x \in E$ and $\varphi \in H,\langle\varphi(\cdot), R(\cdot, x)\rangle=\varphi(x)$.

Remark 2. Condition (2) in Definition 1 is called "the reproducing property," which means that the value of the function $\varphi$ at the point $x$ is reproducing by the inner product of $\varphi(\cdot)$ with $R(\cdot, x)$. A Hilbert space which possesses a reproducing kernel is called a RKHS.

To solve (1) and (2) using RKHS method, we first define and construct a reproducing kernel space $W_{2}^{2}[0,1]$ in which every function satisfies the periodic boundary condition $u(0)=u(1)$. After that, we utilize the reproducing kernel space $W_{2}^{1}[0,1]$.

Definition 3. The inner product space $W_{2}^{2}[0,1]$ is defined as $W_{2}^{2}[0,1]=\left\{u(x) \mid u, u^{\prime}\right.$ are absolutely continuous real-valued functions on $[0,1], u, u^{\prime}, u^{\prime \prime} \in L^{2}[0,1]$, and $\left.u(0)=u(1)\right\}$. On the other hand, the inner product and the norm in $W_{2}^{2}[0,1]$ are defined, respectively, by

$$
\langle u, v\rangle_{W_{2}^{2}}=\sum_{i=0}^{1} u^{(i)}(0) v^{(i)}(0)+\int_{0}^{1} u^{\prime \prime}(t) v^{\prime \prime}(t) d t
$$

and $\|u\|_{W_{2}^{2}}=\sqrt{\langle u, u\rangle_{W_{2}^{2}}}$, where $u, v \in W_{2}^{2}[0,1]$.

It is easy to see that $\langle u, v\rangle_{W_{2}^{2}}$ satisfies all the requirements for the inner product. First, $\langle u, u\rangle_{W_{2}^{2}} \geq 0$. Second, $\langle u, v\rangle_{W_{2}^{2}}=\langle v, u\rangle_{W_{2}^{2}}$. Third, $\langle\gamma u, v\rangle_{W_{2}^{2}}=\gamma\langle u, v\rangle_{W_{2}^{2}}$. Fourth, $\langle u+w, v\rangle_{W_{2}^{2}}=\langle u, v\rangle_{W_{2}^{2}}+\langle w, v\rangle_{W_{2}^{2}}$, where $u, v, w \in W_{2}^{2}[0,1]$. It therefore remains only to prove that $\langle u, u\rangle_{W_{2}^{2}}=0$ if and only if $u=0$. In fact, it is obvious that when $u=0$, then $\langle u, u\rangle_{W_{2}^{2}}=0$. On the other hand, if $\langle u, u\rangle_{W_{2}^{2}}=0$, then by (3), we have $\langle u, u\rangle_{W_{2}^{2}}=\sum_{i=0}^{1}\left(u^{(i)}(0)\right)^{2}+\int_{0}^{1}\left(u^{\prime \prime}(t)\right)^{2} d t=0$; therefore, $u(0)=u^{\prime}(0)=0$ and $u^{\prime \prime}(t)=0$. Then, we can obtain $u=0$.

Definition 4 (see [23]). The Hilbert space $W_{2}^{2}[0,1]$ is called a reproducing kernel if, for each fixed $x \in[0,1]$, there exist $R(x, y) \in W_{2}^{2}[0,1]$ (simply $R_{x}(y)$ ) such that $\left\langle u(y), R_{x}(y)\right\rangle_{W_{2}^{2}}=u(x)$ for any $u(y) \in W_{2}^{2}[0,1]$ and $y \in$ $[0,1]$.

An important subset of the RKHSs is the RKHSs associated with continuous kernel functions. These spaces have wide applications, including complex analysis, harmonic analysis, quantum mechanics, statistics, and machine learning.

Theorem 5. The Hilbert space $W_{2}^{2}[0,1]$ is a complete reproducing kernel and its reproducing kernel function $R_{x}(y)$ can be written as

$$
\begin{aligned}
& R_{x}(y) \\
& = \begin{cases}p_{1}(x)+p_{2}(x) y+p_{3}(x) y^{2}+p_{4}(x) y^{3}, & y \leq x, \\
q_{1}(x)+q_{2}(x) y+q_{3}(x) y^{2}+q_{4}(x) y^{3}, & y>x,\end{cases}
\end{aligned}
$$


where $p_{i}(x)$ and $q_{i}(x), i=1,2,3,4$, are unknown coefficients of $R_{x}(y)$ and will be given in the following proof.

Proof. The proof of the completeness and reproducing property of $W_{2}^{2}[0,1]$ is similar to the proof in [24]. Now, let us find out the expression form of the reproing kernel function $R_{x}(y)$ in the space $W_{2}^{2}[0,1]$. Through several integration by parts, we have $\int_{0}^{1} u^{\prime \prime}(y) \partial_{y}^{3} R_{x}(y) d y=\left.\sum_{i=0}^{1}(-1)^{1-i} u^{(i)}(y) \partial_{y}^{3-i} R_{x}(y)\right|_{y=0} ^{y=1}+$ $\int_{0}^{1} u(y) \partial_{y}^{4} R_{x}(y) d y$. Thus, from (3), we can write $\left\langle u(y), R_{x}(y)\right\rangle_{W_{2}^{2}}=\sum_{i=0}^{1} u^{(i)}(0)\left[\partial_{y}^{i} R_{x}(0)+(-1)^{i} \partial_{y}^{3-i} R_{x}(0)\right]$ $+\sum_{i=0}^{1}(-1)^{1-i} u^{(i)}(1) \partial_{y}^{3-i} R_{x}(1)+\int_{0}^{1} u(y) \partial_{y}^{4} R_{x}(y) d y$. Since $R_{x}(y) \in W_{2}^{2}[0,1]$, it follows that $R_{x}(0)=R_{x}(1)$; also since $u(x) \in W_{2}^{2}[0,1]$, it follows that $u(0)=u(1)$. Then

$$
\begin{aligned}
& \left\langle u(y), R_{x}(y)\right\rangle_{W_{2}^{2}} \\
& =\sum_{i=0}^{1} u^{(i)}(0)\left[\partial_{y}^{i} R_{x}(0)+(-1)^{i} \partial_{y}^{3-i} R_{x}(0)\right] \\
& \quad+\sum_{i=0}^{1}(-1)^{i+1} u^{(i)}(1) \partial_{y}^{3-i} R_{x}(1) \\
& \quad+\int_{0}^{1} u(y) \partial_{y}^{4} R_{x}(y) d y+c_{1}(u(0)-u(1)) .
\end{aligned}
$$

But on the other aspect as well, if $\partial_{y}^{2} R_{x}(1)=0$, $R_{x}(0)+\partial_{y}^{3} R_{x}(0)+c_{1}=0, \partial_{y}^{1} R_{x}(0)-\partial_{y}^{2} R_{x}(0)=0$, and $\partial_{y}^{3} R_{x}(1)+c_{1}=0$, then (5) implies that $\left\langle u(y), R_{x}(y)\right\rangle_{W_{2}^{2}}=$ $\int_{0}^{1} u(y) \partial_{y}^{4} R_{x}(y) d y$. Now, for any $x \in[0,1]$, if $R_{x}(y)$ satisfies

$$
\partial_{y}^{4} R_{x}(y)=-\delta(x-y), \quad \delta \text { dirac-delta function, }
$$

then $\left\langle u(y), R_{x}(y)\right\rangle_{W_{2}^{2}}=u(x)$. Obviously, $R_{x}(y)$ is the reproducing kernel function of the space $W_{2}^{2}[0,1]$. Next, we give the expression form of the reproducing kernel function $R_{x}(y)$. The characteristic formula of (6) is given by $\lambda^{4}=0$. Then the characteristic values are $\lambda=0$ with multiplicity 4. So, let the expression form of the reproducing kernel function $R_{x}(y)$ be as defined in (4). On the other hand, for (6), let $R_{x}(y)$ satisfy the equation $\partial_{y}^{m} R_{x}(x+0)=\partial_{y}^{m} R_{x}(x-0)$, $m=0,1,2$. Integrating $\partial_{y}^{6} R_{x}(y)=-\delta(x-y)$ from $x-\varepsilon$ to $x+\varepsilon$ with respect to $y$ and letting $\varepsilon \rightarrow 0$, we have the jump degree of $\partial_{y}^{5} R_{x}(y)$ at $y=x$ given by $\partial_{y}^{3} R_{x}(x+0)-\partial_{y}^{3} R_{x}(x-0)=-1$. Through the last descriptions, the unknown coefficients of (4) can be obtained. However, by using MAPLE 13 software package, the representation form of the reproducing kernel function $R_{x}(y)$ is provided by

$$
R_{x}(y)= \begin{cases}\frac{1}{48}\left(x^{3} y\left(6+3 y-y^{2}\right)+3 x^{2} y\left(-6-3 y y^{2}\right)+6 x y\left(2+y+y^{2}\right)-8\left(-6+y^{3}\right)\right), & y \leq x \\ \frac{1}{48}\left(48+6 x y\left(2-3 y+y^{2}\right)+3 x^{2} y\left(2-3 y+y^{2}\right)-x^{3}\left(8-6 y-3 y^{2}+y^{3}\right)\right), & y>x\end{cases}
$$

This completes the proof.

Definition 6 (see [25]). The inner product space $W_{2}^{1}[0,1]$ is defined as $W_{2}^{1}[0,1]=\{u(x) \mid u$ is absolutely continuous realvalued function on $[0,1]$ and $\left.u^{\prime} \in L^{2}[0,1]\right\}$. On the other hand, the inner product and the norm in $W_{2}^{1}[0,1]$ are defined, respectively, by $\langle u(x), v(x)\rangle_{W_{2}^{1}}=u(0) v(0)+\int_{0}^{1} u^{\prime}(x) v^{\prime}(x) d x$, and $\|u\|_{W_{2}^{1}}=\sqrt{\langle u, u\rangle_{W_{2}^{1}}}$, where $u, v \in W_{2}^{1}[0,1]$.

Theorem 7 (see [25]). The Hilbert space $W_{2}^{1}[0,1]$ is a complete reproducing kernel and its reproducing kernel function $G_{x}(y)$ can be written as

$$
G_{x}(y)= \begin{cases}1+y, & y \leq x \\ 1+x, & y>x\end{cases}
$$

Reproducing kernel functions possess some important properties such as being symmetric, unique, and nonnegative. The reader is asked to refer to [23-35] in order to know more details about reproducing kernel functions, including their mathematical and geometrical properties, their types and kinds, and their applications and method of calculations.

\section{Formulation of Linear Operator}

In this section, the formulation of a differential linear operator and the implementation method are presented in the reproducing kernel space $W_{2}^{2}[0,1]$. After that, we construct an orthogonal function system of the space $W_{2}^{2}[0,1]$ based on the use of the Gram-Schmidt orthogonalization process in order to obtain the exact and approximate solutions of (1) and (2) using RKHS method.

First, as in [23-35], we transform the problem into a differential operator. To do this, we define a differential operator $L$ as $L: W_{2}^{2}[0,1] \rightarrow W_{2}^{1}[0,1]$ such that $L u(x)=$ $u^{\prime}(x)$. As a result, (1) and (2) can be converted into the equivalent form as follows:

$$
\begin{gathered}
L u_{s}(x)=F_{s}\left(x, u_{1}(x), u_{2}(x), \ldots, u_{n}(x)\right), \\
u_{s}(0)-u_{s}(1)=0,
\end{gathered}
$$

where $0 \leq x \leq 1$ and $s=1,2, \ldots, n$ in which $u_{s}(x) \in W_{2}^{2}[0,1]$ and $F_{s}\left(x, v_{1}, v_{1}, \ldots, v_{n}\right) \in W_{2}^{1}[0,1]$ for $v_{s}=v_{s}(x) \in W_{2}^{2}[0,1]$, $-\infty<v_{s}<\infty$, and $0 \leq x \leq 1$. It is easy to show that $L$ is 
a bounded linear operator from the space $W_{2}^{2}[0,1]$ into the space $W_{2}^{1}[0,1]$.

Initially, we construct an orthogonal function system of $W_{2}^{2}[0,1]$. To do so, put $\varphi_{i}(x)=G_{x_{i}}(x)$ and $\psi_{i}(x)=L^{*} \varphi_{i}(x)$, where $\left\{x_{i}\right\}_{i=1}^{\infty}$ is dense on $[0,1]$ and $L^{*}$ is the adjoint operator of $L$. In terms of the properties of reproducing kernel function $G_{x}(y)$, one obtains $\left\langle u_{s}(x), \psi_{i}(x)\right\rangle_{W_{2}^{2}}=\left\langle u_{s}(x), L^{*} \varphi_{i}(x)\right\rangle_{W_{2}^{2}}=$ $\left\langle L u_{s}(x), \varphi_{i}(x)\right\rangle_{W_{2}^{1}}=L u_{s}\left(x_{i}\right), i=1,2, \ldots, s=1,2, \ldots, n$.

For the orthonormal function system $\left\{\bar{\psi}_{i}(x)\right\}_{i=1}^{\infty}$ of the space $W_{2}^{2}[0,1]$, it can be derived from the Gram-Schmidt orthogonalization process of $\left\{\psi_{i}(x)\right\}_{i=1}^{\infty}$ as follows:

$$
\bar{\psi}_{i}(x)=\sum_{k=1}^{i} \beta_{i k} \psi_{k}(x),
$$

where $\beta_{i k}$ are orthogonalization coefficients and are given as

$$
\begin{gathered}
\beta_{i j}=\frac{1}{\left\|\psi_{1}\right\|}, \quad \text { for } i=j=1, \\
\beta_{i j}=\frac{1}{\sqrt{\left\|\psi_{i}\right\|^{2}-\sum_{k=1}^{i-1}\left(\left\langle\psi_{i}, \bar{\psi}_{k}\right\rangle_{W_{2}^{2}}\right)^{2}}}, \quad \text { for } i=j \neq 1, \\
\beta_{i j}=-\frac{1}{\sqrt{\left\|\psi_{i}\right\|^{2}-\sum_{k=1}^{i-1}\left(c_{i k}\right)^{2}} \sum_{k=j}^{i-1}\left\langle\psi_{i}, \bar{\psi}_{k}\right\rangle_{W_{2}^{2}} \beta_{k j},}
\end{gathered}
$$$$
\text { for } i>j \text {. }
$$

Clearly, $\psi_{i}(x)=L^{*} \varphi_{i}(x)=\left\langle L^{*} \varphi_{i}(x), R_{x}(y)\right\rangle_{W_{2}^{2}}=$ $\left\langle\varphi_{i}(x), L_{y} R_{x}(y)\right\rangle_{W_{2}^{1}}=\left.L_{y} R_{x}(y)\right|_{y=x_{i}} \in W_{2}^{2}[0,1]$. Thus, $\psi_{i}(x)$ can be written in the form $\psi_{i}(x)=\left.L_{y} R_{x}(y)\right|_{y=x_{i}}$, where $L_{y}$ indicates that the operator $L$ applies to the function of $y$.

Theorem 8. If $\left\{x_{i}\right\}_{i=1}^{\infty}$ is dense on $[0,1]$, then $\left\{\psi_{i}(x)\right\}_{i=1}^{\infty}$ is a complete function system of the space $W_{2}^{2}[0,1]$.

Proof. For each fixed $u_{s}(x) \in W_{2}^{2}[0,1]$, let $\left\langle u_{s}(x), \psi_{i}(x)\right\rangle_{W_{2}^{2}}=$ 0 . In other words, one can write $\left\langle u_{s}(x), \psi_{i}(x)\right\rangle_{W_{2}^{2}}=$ $\left\langle u_{s}(x), L^{*} \varphi_{i}(x)\right\rangle_{W_{2}^{2}}=\left\langle L u_{s}(x), \varphi_{i}(x)\right\rangle_{W_{2}^{1}}=L u_{s}\left(x_{i}\right)=0$. Note that $\left\{x_{i}\right\}_{i=1}^{\infty}$ is dense on $[0,1]$; therefore $L u_{s}(x)=0$. It follows that $u_{s}(x)=0, s=1,2, \ldots, n$, from the existence of $L^{-1}$. So, the proof of the theorem is complete.

Lemma 9. If $u_{s}(x) \in W_{2}^{2}[0,1]$, then there exist positive constants $M^{\{s\}}$ such that $\left\|u_{s}^{(i)}(x)\right\|_{C} \leq M^{\{s\}}\left\|u_{s}(x)\right\|_{W_{2}^{2}}, i=0,1$, $s=1,2, \ldots, n$, where $\left\|u_{s}(x)\right\|_{C}=\max _{0 \leq x \leq 1}\left|u_{s}(x)\right|$.

Proof. For any $x, y \in[0,1]$, we have $u_{s}^{(i)}(x)=$ $\left\langle u_{s}(y), \partial_{x}^{i} R_{x}(y)\right\rangle_{W_{2}^{2}}$. By the expression form of the kernel function $R_{x}(y)$, it follows that $\left\|\partial_{x}^{i} R_{x}(y)\right\|_{W_{2}^{2}} \leq M_{i}^{\{s\}}$. Thus, $\left|u_{s}^{(i)}(x)\right|=\left|\left\langle u_{s}(x), \partial_{x}^{i} R_{x}(x)\right\rangle_{W_{2}^{2}}\right| \leq\left\|\partial_{x}^{i} R_{x}(x)\right\|_{W_{2}^{2}}\left\|u_{s}(x)\right\|_{W_{2}^{2}} \leq$ $M_{i}^{\{s\}}\left\|u_{s}(x)\right\|_{W_{2}^{2}}$. Hence, $\left\|u_{s}^{(i)}(x)\right\|_{C} \leq \max _{i=0,1}\left\{M_{i}^{\{s\}}\right\}\left\|u_{s}(x)\right\|_{W_{2}^{2}}$, $i=0,1, s=1,2, \ldots, n$.
The internal structure of the following theorem is as follows: firstly, we will give the representation form of the exact solutions of (1) and (2) in the form of an infinite series in the space $W_{2}^{2}[0,1]$. After that, the convergence of approximate solutions $u_{s, m}(x)$ to the exact solutions $u_{s}(x)$, $s=1,2, \ldots, n$, will be proved.

Theorem 10. For each $u_{s}, s=1,2, \ldots, n$ in the space $W_{2}^{2}[0,1]$, the series $\sum_{i=1}^{\infty}\left\langle u_{s}(x), \bar{\psi}_{i}(x)\right\rangle \bar{\psi}_{i}(x)$ is convergent in the sense of the norm of $W_{2}^{2}[0,1]$. On the other hand, if $\left\{x_{i}\right\}_{i=1}^{\infty}$ is dense on $[0,1]$, then the following hold:

(i) the exact solutions of (9) could be represented by

$$
\begin{aligned}
& u_{s}(x) \\
& =\sum_{i=1}^{\infty} \sum_{k=1}^{i} \beta_{i k} F_{s}\left(x_{k}, u_{1}\left(x_{k}\right), u_{2}\left(x_{k}\right), \ldots, u_{n}\left(x_{k}\right)\right) \bar{\psi}_{i}(x),
\end{aligned}
$$

(ii) the approximate solutions of (9)

$$
\begin{aligned}
& u_{s, m}(x) \\
& =\sum_{i=1}^{m} \sum_{k=1}^{i} \beta_{i k} F_{s}\left(x_{k}, u_{1}\left(x_{k}\right), u_{2}\left(x_{k}\right), \ldots, u_{n}\left(x_{k}\right)\right) \bar{\psi}_{i}(x),
\end{aligned}
$$

and $u_{s, m}^{(i)}(x), i=0,1$, are converging uniformly to the exact solutions $u_{s}(x)$ and their derivatives as $m \rightarrow \infty$, respectively.

Proof. For the first part, let $u_{s}(x)$ be solutions of (9) in the space $W_{2}^{2}[0,1]$. Since $u_{s}(x) \in W_{2}^{2}[0,1]$, $\sum_{i=1}^{\infty}\left\langle u_{s}(x), \bar{\psi}_{i}(x)\right\rangle \bar{\psi}_{i}(x)$ is the Fourier series expansion about normal orthogonal system $\left\{\bar{\psi}_{i}(x)\right\}_{i=1}^{\infty}$, and $W_{2}^{2}[0,1]$ is the Hilbert space, then the series $\sum_{i=1}^{\infty}\left\langle u_{s}(x), \bar{\psi}_{i}(x)\right\rangle \bar{\psi}_{i}(x)$ is convergent in the sense of $\|\cdot\|_{W_{2}^{2}}$. On the other hand, using (10), it easy to see that

$$
\begin{aligned}
u_{s}(x) & =\sum_{i=1}^{\infty}\left\langle u_{s}(x), \bar{\psi}_{i}(x)\right\rangle_{W_{2}^{2}} \bar{\psi}_{i}(x) \\
& =\sum_{i=1}^{\infty} \sum_{k=1}^{i} \beta_{i k}\left\langle u_{s}(x), \psi_{k}(x)\right\rangle_{W_{2}^{2}} \bar{\psi}_{i}(x) \\
& =\sum_{i=1}^{\infty} \sum_{k=1}^{i} \beta_{i k}\left\langle u_{s}(x), L^{*} \varphi_{k}(x)\right\rangle_{W_{2}^{2}} \bar{\psi}_{i}(x) \\
& =\sum_{i=1}^{\infty} \sum_{k=1}^{i} \beta_{i k}\left\langle L u_{s}(x), \varphi_{k}(x)\right\rangle_{W_{2}^{1}} \bar{\psi}_{i}(x)
\end{aligned}
$$




$$
\begin{gathered}
=\sum_{i=1}^{\infty} \sum_{k=1}^{i} \beta_{i k}\left\langle F_{s}\left(x, u_{1}(x), u_{2}(x), \ldots, u_{n}(x)\right),\right. \\
\left.\varphi_{k}(x)\right\rangle_{W_{2}^{1}} \bar{\psi}_{i}(x) \\
=\sum_{i=1}^{\infty} \sum_{k=1}^{i} \beta_{i k} F_{s}\left(x_{k}, u_{1}\left(x_{k}\right), u_{2}\left(x_{k}\right), \ldots,\right. \\
\left.u_{n}\left(x_{k}\right)\right) \bar{\psi}_{i}(x) .
\end{gathered}
$$

Therefore, the form of (12) is the exact solutions of (9). For the second part, it is easy to see that by Lemma 9 , for any $x \in$ $[0,1]$,

$$
\begin{aligned}
& \left|u_{s, m}^{(i)}(x)-u_{s}^{(i)}(x)\right| \\
& \quad=\left|\left\langle u_{s, m}(x)-u_{s}(x), R_{x}^{(i)}(x)\right\rangle_{W_{2}^{2}}\right| \\
& \quad \leq\left\|\partial_{x}^{i} R_{x}(x)\right\|_{W_{2}^{2}}\left\|u_{s, m}(x)-u_{s}(x)\right\|_{W_{2}^{2}} \\
& \quad \leq M_{i}^{\{s\}}\left\|u_{s, m}(x)-u_{s}(x)\right\|_{W_{2}^{2}},
\end{aligned}
$$

where $i=0,1$ and $M_{i}^{\{s\}}$ are positive constants. Hence, if $\left\|u_{s, m}(x)-u_{s}(x)\right\|_{W_{2}^{2}} \rightarrow 0$ as $m \rightarrow \infty$, the approximate solutions $u_{s, m}(x)$ and $u_{s, m}^{(i)}(x), i=0,1, s=1,2, \ldots, n$, are converged uniformly to the exact solutions $u_{s}(x)$ and their derivatives, respectively. So, the proof of the theorem is complete.

We mention here that the approximate solutions $u_{s, m}(x)$ in (13) can be obtained directly by taking finitely many terms in the series representation for $u_{s}(x)$ of (12).

\section{Construction of Iterative Method}

In this section, an iterative method of obtaining the solutions of (1) and (2) is represented in the reproducing kernel space $W_{2}^{2}[0,1]$ for linear and nonlinear cases. Initially, we will mention the following remark about the exact and approximate solutions of (1) and (2).

In order to apply the RKHS technique to solve (1) and (2), we have the following two cases based on the algebraic structure of the function $F_{s}, s=1,2, \ldots, n$.

Case 1. If (1) is linear, then the exact and approximate solutions can be obtained directly from (12) and (13), respectively.

Case 2. If (1) is nonlinear, then in this case the exact and approximate solutions can be obtained by using the following iterative algorithm.

Algorithm 11. According to (12), the representation form of the solutions of (1) can be denoted by

$$
u_{s}(x)=\sum_{i=1}^{\infty} B_{i}^{\{s\}} \bar{\psi}_{i}(x), \quad s=1,2, \ldots, n,
$$

where $B_{i}^{\{s\}}=\sum_{k=1}^{i} \beta_{i k} F_{s}\left(x_{k}, u_{1, k-1}\left(x_{k}\right), u_{2, k-1}\left(x_{k}\right), \ldots\right.$, $u_{n, k-1}\left(x_{k}\right)$ ). In fact, $B_{i}^{\{s\}}$ in (16) are unknown; one will approximate $B_{i}^{\{s\}}$ using known $A_{i}^{\{s\}}$. For numerical computations, one defines the initial functions $u_{s, 0}\left(x_{1}\right)=0$, put $u_{s, 0}\left(x_{1}\right)=u_{s}\left(x_{1}\right)$, and define the $m$-term approximations to $u_{s}(x)$ by

$$
u_{s, m}(x)=\sum_{i=1}^{m} A_{i}^{\{s\}} \bar{\psi}_{i}(x), \quad s=1,2, \ldots, n,
$$

where the coefficients $A_{i}^{\{s\}}$ of $\bar{\psi}_{i}(x), i=1,2, \ldots, n, s=$ $1,2, \ldots, n$, are given as

$$
\begin{gathered}
A_{1}^{\{s\}}=\beta_{11} F_{s}\left(x_{1}, u_{1,0}\left(x_{1}\right), u_{2,0}\left(x_{1}\right), \ldots, u_{n, 0}\left(x_{1}\right)\right), \\
u_{s, 1}(x)=A_{1}^{\{s\}} \bar{\psi}_{1}(x), \\
A_{2}^{\{s\}}=\sum_{k=1}^{2} \beta_{2 k} F_{s}\left(x_{k}, u_{1, k-1}\left(x_{k}\right), u_{2, k-1}\left(x_{k}\right), \ldots,\right. \\
\left.u_{n, k-1}\left(x_{k}\right)\right), \\
u_{s, 2}(x)=\sum_{i=1}^{2} A_{i}^{\{s\}} \bar{\psi}_{i}(x), \\
\vdots \\
A_{n}^{\{s\}}=\sum_{k=1}^{m} \beta_{m k} F_{s}\left(x_{k}, u_{1, k-1}\left(x_{k}\right), u_{2, k-1}\left(x_{k}\right), \ldots,\right. \\
\left.u_{n, k-1}\left(x_{k}\right)\right), \\
u_{s, m}(x)=\sum_{i=1}^{m-1} A_{i}^{\{s\}} \bar{\psi}_{i}(x) .
\end{gathered}
$$

Here, we note that, in the iterative process of (17), we can guarantee that the approximations $u_{s, m}(x)$ satisfy the periodic boundary conditions (2). Now, the approximate solutions $u_{s, m}^{M}(x)$ can be obtained by taking finitely many terms in the series representation of $u_{s, m}(x)$ and

$$
\begin{aligned}
& u_{s, m}^{M}(x) \\
& =\sum_{i=1}^{M} \sum_{k=1}^{i} \beta_{i k} F_{s}\left(x_{k}, u_{1, m-1}\left(x_{k}\right), u_{2, m-1}\left(x_{k}\right), \ldots,\right. \\
& \left.u_{n, m-1}\left(x_{k}\right)\right) \bar{\psi}_{i}(x), \\
& s=1,2, \ldots, n .
\end{aligned}
$$

Now, we will proof that $u_{s, m}(x)$ in the iterative formula (17) are converged to the exact solutions $u_{s}(x)$ of (1). In fact, this result is a fundamental in the RKHS theory and its applications. The next two lemmas are collected in order to prove the prerecent theorem.

Lemma 12. If $\left\|u_{s, m}(x)-u_{s}(x)\right\|_{W_{2}^{2}} \rightarrow 0, x_{m} \rightarrow y$ as $m \rightarrow \infty$, and $F_{s}\left(x, v_{1}, v_{2}, \ldots, v_{n}\right)$ is continuous in $[0,1]$ 
with respect to $x, v_{i}$, for $x \in[0,1]$ and $v_{i} \in(-\infty, \infty)$, then $F_{s}\left(x_{m}, u_{1, m-1}\left(x_{m}\right), u_{2, m-1}\left(x_{m}\right), \ldots, u_{n, m-1}\left(x_{m}\right)\right) \quad \rightarrow$ $F_{s}\left(y, u_{1}(y), u_{2}(y), \ldots, u_{n}(y)\right), s=1,2, \ldots, n$ as $m \rightarrow \infty$.

Proof. Firstly, we will prove that $u_{s, m-1}\left(x_{m}\right) \rightarrow u_{s}(y)$ in the sense of $\|\cdot\|_{W_{2}^{2}}$. Since

$$
\begin{aligned}
& \left|u_{s, m-1}\left(x_{m}\right)-u_{s}(y)\right| \\
& \quad=\left|u_{s, m-1}\left(x_{m}\right)-u_{s, m-1}(y)+u_{s, m-1}(y)-u_{s}(y)\right| \\
& \quad \leq\left|u_{s, m-1}\left(x_{m}\right)-u_{s, m-1}(y)\right|+\left|u_{s, m-1}(y)-u_{s}(y)\right| .
\end{aligned}
$$

By reproducing property of $R_{x}(y)$, we have $u_{s, m-1}\left(x_{m}\right)=$ $\left\langle u_{s, m-1}(x), R_{x_{m}}(x)\right\rangle$ and $u_{s, m-1}(y)=\left\langle u_{s, m-1}(x), R_{y}(x)\right\rangle$. Thus, $\left|u_{s, m-1}\left(x_{m}\right)-u_{s, m-1}(y)\right|=\left|\left\langle u_{s, m-1}(x), R_{x_{m}}(x)-R_{y}(x)\right\rangle_{W_{2}^{2}}\right| \leq$ $\left\|u_{s, m-1}(x)\right\|_{W_{2}^{2}}\left\|R_{x_{m}}(x)-R_{y}(x)\right\|_{W_{2}^{2}}$. From the symmetry of $R_{x}(y)$, it follows that $\left\|R_{x_{m}}(x)-R_{y}(x)\right\|_{W_{2}^{2}} \rightarrow 0$ as $m \rightarrow \infty$. Hence, $\left|u_{s, m-1}\left(x_{m}\right)-u_{s, m-1}(y)\right| \stackrel{\rightarrow}{\rightarrow} 0$ as soon as $x_{m} \rightarrow y$. On the other hand, by Theorem 10 part (ii), for any $y \in[0,1]$, it holds that $\left|u_{s, m-1}(y)-u_{s}(y)\right| \rightarrow 0$ as $m \rightarrow \infty$. Therefore, $u_{s, m-1}\left(x_{m}\right) \rightarrow u_{s}(y)$ in the sense of $\|\cdot\|_{W_{2}^{2}}$ as $x_{m} \rightarrow y$ and $m \rightarrow \infty$. Thus, by means of the continuation of $F_{s}$, it is obtained that $F_{s}\left(x_{m}, u_{1, m-1}\left(x_{m}\right), u_{2, m-1}\left(x_{m}\right), \ldots, u_{n, m-1}\left(x_{m}\right)\right)$ $F_{s}\left(y, u_{1}(y), u_{2}(y), \ldots, u_{n}(y)\right), s=1,2, \ldots, n$ as $m \rightarrow \infty$.

Lemma 13. For $j \leq m$, one has $L u_{s, m}\left(x_{j}\right)=L u_{s}\left(x_{j}\right)=$ $F_{s}\left(x_{j}, u_{1, j-1}\left(x_{j}\right), u_{2, j-1}\left(x_{j}\right), \ldots, u_{n, j-1}\left(x_{j}\right)\right), s=1,2, \ldots, n$.

Proof. The proof of $L u_{s, m}\left(x_{j}\right)=F_{s}\left(x_{j}, u_{1, j-1}\left(x_{j}\right)\right.$, $\left.u_{2, j-1}\left(x_{j}\right), \ldots, u_{n, j-1}\left(x_{j}\right)\right)$ will be obtained by induction as follows: if $j \leq m$, then $L u_{s, m}\left(x_{j}\right)=\sum_{i=1}^{m} A_{i}^{\{s\}} L \bar{\psi}_{i}\left(x_{j}\right)=$ $\sum_{i=1}^{m} A_{i}^{\{s\}}\left\langle L \bar{\psi}_{i}(x), \varphi_{j}(x)\right\rangle_{W_{2}^{1}}=\sum_{i=1}^{m} A_{i}^{\{s\}}\left\langle\bar{\psi}_{i}(x), L_{j}^{*} \varphi(x)\right\rangle_{W_{2}^{2}}$ $=\sum_{i=1}^{m} A_{i}^{\{s\}}\left\langle\bar{\psi}_{i}(x), \psi_{j}(x)\right\rangle_{W_{2}^{2}}$. Using the orthogonality of $\left\{\bar{\psi}_{i}(x)\right\}_{i=1}^{\infty}$, it yields that

$$
\begin{aligned}
& \sum_{l=1}^{j} \beta_{j l} L u_{s, m}\left(x_{l}\right) \\
& \quad=\sum_{i=1}^{m} A_{i}^{\{s\}}\left\langle\bar{\psi}_{i}(x), \sum_{l=1}^{j} \beta_{j l} \psi_{l}(x)\right\rangle_{W_{2}^{2}} \\
& \quad=\sum_{i=1}^{m} A_{i}^{\{s\}}\left\langle\bar{\psi}_{i}(x), \bar{\psi}_{j}(x)\right\rangle_{W_{2}^{2}}=A_{j}^{\{s\}} \\
& \quad=\sum_{l=1}^{j} \beta_{j l} F_{s}\left(x_{l}, u_{1, l-1}\left(x_{l}\right), u_{2, l-1}\left(x_{l}\right), \ldots, u_{n, l-1}\left(x_{l}\right)\right) .
\end{aligned}
$$

Now, if $j=1$, then $L u_{s, m}\left(x_{1}\right)=F_{s}\left(x_{1}, u_{1,0}\left(x_{1}\right), u_{2,0}\left(x_{1}\right)\right.$, $\left.\ldots, u_{n, 0}\left(x_{1}\right)\right)$. Again, if $j=2$, then $\beta_{21} L u_{s, m}\left(x_{1}\right)+$ $\beta_{22} L u_{s, m}\left(x_{2}\right)=\beta_{21} F_{s}\left(x_{1}, u_{1,0}\left(x_{1}\right), u_{2,0}\left(x_{1}\right), \ldots, u_{n, 0}\left(x_{1}\right)\right)+$ $\beta_{22} F_{s}\left(x_{2}, u_{1,1}\left(x_{2}\right), u_{2,1}\left(x_{2}\right), \ldots, u_{n, 1}\left(x_{2}\right)\right)$. Thus, $L u_{s, m}\left(x_{2}\right)=$ $F_{s}\left(x_{2}, u_{1,1}\left(x_{2}\right), u_{2,1}\left(x_{2}\right), \ldots, u_{n, 1}\left(x_{2}\right)\right)$. Indeed, it is easy to see by using mathematical induction that $L u_{s, m}\left(x_{j}\right)=$
$F_{s}\left(x_{j}, u_{1, j-1}\left(x_{j}\right), u_{2, j-1}\left(x_{j}\right), \ldots, u_{n, j-1}\left(x_{j}\right)\right), s=1,2, \ldots, n$. But on the other hand, from Theorem 10, $u_{s, m}(x)$ converge uniformly to $u_{s}(x)$. It follows that, on taking limits in (17), $u_{s}(x)=\sum_{i=1}^{\infty} A_{i}^{\{s\}} \bar{\psi}_{i}(x)$. Therefore, $u_{s, m}(x)=P_{m} u_{s}(x)$, where $P_{m}$ is an orthogonal projector from the space $W_{2}^{2}[0,1]$ to Span $\left\{\psi_{1}, \psi_{2}, \ldots, \psi_{m}\right\}$. Thus,

$$
\begin{aligned}
& L u_{s, m}\left(x_{j}\right) \\
& \quad=\left\langle L u_{s, m}(x), \varphi_{j}(x)\right\rangle_{W_{2}^{1}}=\left\langle u_{s, m}(x), L_{j}^{*} \varphi(x)\right\rangle_{W_{2}^{2}} \\
& \quad=\left\langle P_{m} u_{s}(x), \psi_{j}(x)\right\rangle_{W_{2}^{2}}=\left\langle u_{s}(x), P_{m} \psi_{j}(x)\right\rangle_{W_{2}^{2}} \\
& =\left\langle u_{s}(x), \psi_{j}(x)\right\rangle_{W_{2}^{2}}=\left\langle L u_{s}(x), \varphi_{j}(x)\right\rangle_{W_{2}^{1}}=L u_{s}\left(x_{j}\right),
\end{aligned}
$$

as $j \leq m$ and $s=1,2, \ldots, n$.

Theorem 14. If $\left\|u_{s, m}\right\|_{W_{2}^{2}}$ is bounded and $\left\{x_{i}\right\}_{i=1}^{\infty}$ is dense on $[0,1]$, then the $m$-term approximate solutions $u_{s, m}(x)$ in the iterative formula (17) converge to the exact solutions $u_{s}(x)$ of (9) in the space $W_{2}^{2}[0,1]$ and $u_{s}(x)=\sum_{i=1}^{\infty} A_{i}^{\{s\}} \bar{\psi}_{i}(x), s=$ $1,2, \ldots, n$, where $A_{i}^{\{s\}}$ is given by (18).

Proof. The proof consists of the following three steps. Firstly, we will prove that the sequence $\left\{u_{s, m}\right\}_{m=1}^{\infty}$ in (17) is monotone increasing in the sense of $\|\cdot\|_{W_{2}^{2}}$. By Theorem 8 , $\left\{\bar{\psi}_{i}\right\}_{i=1}^{\infty}$ is the complete orthonormal system in the space $W_{2}^{2}[0,1]$. Hence, we have $\left\|u_{s, m}\right\|_{W_{2}^{2}}^{2}=\left\langle u_{s, m}(x), u_{s, m}(x)\right\rangle_{W_{2}^{2}}=$ $\left\langle\sum_{i=1}^{m} A_{i}^{\{s\}} \bar{\psi}_{i}(x), \sum_{i=1}^{m} A_{i}^{\{s\}} \bar{\psi}_{i}(x)\right\rangle_{W_{2}^{2}}=\sum_{i=1}^{m}\left(A_{i}^{\{s\}}\right)^{2}$. Therefore, $\left\|u_{s, m}\right\|_{W_{2}^{2}}, s=1,2, \ldots, n$, is monotone increasing. Secondly, we will prove the convergence of $u_{s, m}(x)$. From (17), we have $u_{s, m+1}(x)=u_{s, m}(x)+A_{m+1}^{\{s\}} \bar{\psi}_{m+1}(x)$. From the orthogonality of $\left\{\bar{\psi}_{i}(x)\right\}_{i=1}^{\infty}$, it follows that $\left\|u_{s, m+1}\right\|_{W_{2}^{2}}^{2}=$ $\left\|u_{s, m}\right\|_{W_{2}^{2}}^{2}+\left(A_{m+1}^{\{s\}}\right)^{2}=\left\|u_{s, m-1}\right\|_{W_{2}^{2}}^{2}+\left(A_{m}^{\{s\}}\right)^{2}+\left(A_{m+1}^{\{s\}}\right)^{2}=$ $\cdots=\left\|u_{s, 0}\right\|_{W_{2}^{2}}^{2}+\sum_{i=1}^{m+1}\left(A_{i}^{\{s\}}\right)^{2}$. Since, the sequence $\left\{u_{s, m}\right\}_{m=1}^{\infty}$ is monotone increasing in the sense of $\|\cdot\|_{W_{2}^{2}}$. Due to the condition that $\left\|u_{s, m}\right\|_{W_{2}^{2}}$ is bounded, $\left\|u_{s, m}\right\|_{W_{2}^{2}}^{2}$ is convergent as $m \rightarrow \infty$. Then, there exist constants $c^{\{s\}}$ such that $\sum_{i=1}^{\infty}\left(A_{i}^{\{s\}}\right)^{2}=c^{\{s\}}$. It implies that $A_{i}^{\{s\}}=$ $\sum_{k=1}^{i} \beta_{i k} F_{s}\left(x_{k}, u_{1, k-1}\left(x_{k}\right), u_{2, k-1}\left(x_{k}\right), \ldots, u_{n, k-1}\left(x_{k}\right)\right) \in l^{2}, i=$ $1,2, \ldots$. On the other hand, since $\left(u_{s, m}-u_{s, m-1}\right) \perp\left(u_{s, m-1}-\right.$ $\left.u_{s, m-2}\right) \perp \cdots \perp\left(u_{s, m+1}-u_{s, m}\right)$ it follows for $l>m$ that

$$
\begin{gathered}
\left\|u_{s, l}(x)-u_{s, m}(x)\right\|_{W_{2}^{2}}^{2} \\
=\| u_{s, l}(x)-u_{s, l-1}(x)+u_{s, l-1}(x)-\cdots \\
+u_{s, l+1}(x)-u_{s, m}(x) \|_{W_{2}^{2}}^{2} \\
=\left\|u_{s, l}(x)-u_{s, l-1}(x)\right\|_{W_{2}^{2}}^{2}+\cdots \\
+\left\|u_{s, l+1}(x)-u_{s, m}(x)\right\|_{W_{2}^{2}}^{2} .
\end{gathered}
$$


Furthermore, $\left\|u_{s, l}(x)-u_{s, l-1}(x)\right\|_{W_{2}^{2}}^{2}=\left(A_{l}^{\{s\}}\right)^{2}$. Consequently, as $l, m \rightarrow \infty$, we have $\left\|u_{s, l}(x)-u_{s, m}(x)\right\|_{W_{2}^{2}}^{2}=$ $\sum_{i=m+1}^{l}\left(A_{i}^{\{s\}}\right)^{2} \rightarrow 0$. Considering the completeness of $W_{2}^{2}[0,1]$, there exists $u_{s}(x) \in W_{2}^{2}[0,1]$ such that $u_{s, l}(x) \rightarrow$ $u_{s}(x), s=1,2, \ldots, n$ as $l \rightarrow \infty$ in the sense of $\|\cdot\|_{W_{2}^{2}}$. Thirdly, we will prove that $u_{s}(x)$ are the solutions of (9). Since $\left\{x_{i}\right\}_{i=1}^{\infty}$ is dense on $[0,1]$, for any $x \in[0,1]$, there exists subsequence $\left\{x_{m_{j}}\right\}_{j=1}^{\infty}$, such that $x_{m_{j}} \rightarrow x$ as $j \rightarrow \infty$. From Lemma 13, it is clear that $L u_{s}\left(x_{m_{j}}\right)=$ $F_{s}\left(x_{m_{j}}, u_{1, m_{j}-1}\left(x_{k}\right), u_{2, m_{j}-1}\left(x_{k}\right), \ldots, u_{n, m_{j}-1}\left(x_{k}\right)\right)$. Hence, let $j \rightarrow \infty$; by Lemma 12 and the continuity of $F_{s}$, we have $L u_{s}(x)=F_{s}\left(x, u_{1}(x), u_{2}(x), \ldots, u_{n}(x)\right)$. That is, $u_{s}(x)$ satisfies (1). Also, since $\bar{\psi}_{i}(x) \in W_{2}^{2}[0,1]$, clearly, $u_{s}(x)$ satisfies the periodic boundary conditions (2). In other words, $u_{s}(x)$ are the solutions of (1) and (2), where $u_{s}(x)=\sum_{i=1}^{\infty} A_{i}^{\{s\}} \bar{\psi}_{i}(x)$ and $A_{i}^{\{s\}}$ are given by (18). The proof is complete.

According to the internal structure of the present method, it is obvious that if we let $u_{s}(x)$ denote the exact solutions of $(9), u_{s, m}(x)$ denote the approximate solutions obtained by the RKHS method as given by (17), and $r_{m}^{\{s\}}(x)$ denote the difference between $u_{s, m}(x)$ and $u_{s}(x)$, where $x \in[0,1]$ and $s=1,2, \ldots, n$, then $\left\|r_{m}^{\{s\}}(x)\right\|_{W_{2}^{2}}^{2}=\left\|u_{s}(x)-u_{s, m}(x)\right\|_{W_{2}^{2}}^{2}=$ $\left\|\sum_{i=m+1}^{\infty} A_{i}^{\{s\}} \bar{\psi}_{i}(x)\right\|_{W_{2}^{2}}^{2}=\sum_{i=m+1}^{\infty}\left(A_{i}^{\{s\}}\right)^{2}$ and $\left\|r_{m-1}^{\{s\}}(x)\right\|_{W_{2}^{2}}^{2}=$ $\sum_{i=m}^{\infty}\left(A_{i}^{\{s\}}\right)^{2}$ or $\left\|r_{m}^{\{s\}}(x)\right\|_{W_{2}^{2}} \leq\left\|r_{m-1}^{\{s\}}(x)\right\|_{W_{2}^{2}}$. Consequently, this shows the following theorem.

Theorem 15. The difference $r_{m}^{\{s\}}(x), s=1,2, \ldots, n$, is monotone decreasing in the sense of the norm of $W_{2}^{2}[0,1]$.

\section{Numerical Examples}

In this section, the theoretical results of the previous sections are illustrated by means of some numerical examples in order to illustrate the performance of the RKHS method for solving systems of first-order periodic BVPs and justify the accuracy and efficiency of the method. To do so, we consider the following three nonlinear examples. These examples have been solved by the presented method with different values of $m$ and $M$. Results obtained by the method are compared with the exact solution of each example by computing the absolute and relative errors and are found to be in good agreement with each other. In the process of computation, all experiments were performed in MAPLE 13 software package.

Example 1. Consider the following first-order nonlinear differential system:

$$
\begin{gathered}
u_{1}^{\prime}(x)-u_{1}(x)+\left(u_{2}(x)\right)^{3}=f_{1}(x), \\
u_{2}^{\prime}(x)-\sinh \left(u_{1}(x)\right) u_{2}(x)=f_{2}(x), \\
f_{1}(x)=(x-1)(\cos x-\sin x)+\sin x+e^{3 x(x-1)}, \\
f_{2}(x)=(\sinh (\sin (x)(1-x))+2 x-1) e^{x(x-1)},
\end{gathered}
$$

subject to the periodic boundary conditions

$$
\begin{aligned}
& u_{1}(0)=u_{1}(1), \\
& u_{2}(0)=u_{2}(1) .
\end{aligned}
$$

The exact solutions are $u_{1}(x)=(x-1) \sin (x)$ and $u_{2}(x)=$ $e^{x(x-1)}$.

Using RKHS method, take $x_{i}=(i-1) /(M-1), i=$ $1,2, \ldots, M$, on $[0,1]$. The numerical results at some selected grid points for $M=101$ and $m=3$ are given in Tables 1 and 2 for the dependent variables $u_{1}(x)$ and $u_{2}(x)$, respectively.

The present method enables us to approximate the solutions and their derivatives at every point of the range of integration. Hence, it is possible to pick any point in $[0,1]$ and as well the approximate solutions and their derivatives will be applicable. Next, new numerical results for Example 1 which include the absolute error at some selected gird points in $[0,1]$ for approximating $u_{1}^{\prime}(x)$ and $u_{2}^{\prime}(x)$, where $x_{i}=(i-1) /(M-1)$, $i=1,2, \ldots, M, M=101$, and $m=3$, are given in Table 3 .

Example 2. Consider the following first-order nonlinear differential system:

$$
\begin{gathered}
u_{1}^{\prime}(x)+\sqrt{u_{1}(x)+1} u_{2}(x)=f_{1}(x), \\
v_{2}^{\prime}(x)-u_{1}(x)\left(u_{2}(x)\right)^{2}+\left(u_{2}(x)\right)^{2}=f_{2}(x), \\
f_{1}(x)=\left(x^{4}-2 x^{3}+x^{2}+1\right)^{-1 / 2}+4 x^{3}-6 x^{2}+2 x, \\
f_{2}(x)=-\frac{x^{4}+2 x^{3}-5 x^{2}+2 x-1}{\left(x^{4}-2 x^{3}+x^{2}+1\right)^{2}},
\end{gathered}
$$

subject to the periodic boundary conditions

$$
\begin{aligned}
& u_{1}(0)=u_{1}(1), \\
& u_{2}(0)=u_{2}(1) .
\end{aligned}
$$

The exact solutions are $u_{1}(x)=(x(x-1))^{2}$ and $u_{2}(x)=$ $1 /\left((x(x-1))^{2}+1\right)$.

Using RKHS method, take $x_{i}=(i-1) /(M-1), i=$ $1,2, \ldots, M$, on $[0,1]$. The numerical results at some selected grid points for $M=101$ and $m=3$ are given in Tables 4 and 5 for the dependent variables $u_{1}(x)$ and $u_{2}(x)$, respectively.

Example 3. Consider the following first-order nonlinear differential system:

$$
\begin{gathered}
u_{1}^{\prime}(x)+u_{3}(x) e^{u_{1}(x)}+\left(u_{2}(x)\right)^{2}=f_{1}(x), \\
u_{2}^{\prime}(x)-u_{2}(x) e^{-u_{1}(x)}+\left(u_{3}(x)\right)^{2}=f_{2}(x), \\
u_{3}^{\prime}(x)-u_{1}(x) u_{2}(x) u_{3}(x)=f_{3}(x),
\end{gathered}
$$


TABLE 1: Numerical results of $u_{1}(x)$ for Example 1.

\begin{tabular}{lcccc}
\hline$x$ & Exact solution & Approximate solution & Absolute error & Relative error \\
\hline 0.16 & -0.133827 & -0.13382630119666272 & $9.92359 \times 10^{-7}$ & $7.41522 \times 10^{-6}$ \\
0.32 & -0.213905 & -0.21390423277976867 & $1.02844 \times 10^{-6}$ & $4.80792 \times 10^{-6}$ \\
0.48 & -0.240125 & -0.24012413380235342 & $1.03748 \times 10^{-6}$ & $4.32058 \times 10^{-6}$ \\
0.64 & -0.214990 & -0.21498933621279104 & $1.02268 \times 10^{-6}$ & $4.75685 \times 10^{-6}$ \\
0.80 & -0.143471 & -0.14347022966680445 & $9.88513 \times 10^{-7}$ & $6.88997 \times 10^{-6}$ \\
0.96 & -0.032768 & -0.03276672205464815 & $9.40677 \times 10^{-7}$ & $2.87075 \times 10^{-5}$ \\
\hline
\end{tabular}

TABLE 2: Numerical results of $u_{2}(x)$ for Example 1 .

\begin{tabular}{lcccr}
\hline$x$ & Exact solution & Approximate solution & Absolute error & Relative error \\
\hline 0.16 & 0.874240 & 0.8742398572490666 & $4.41286 \times 10^{-7}$ & $5.04765 \times 10^{-7}$ \\
0.32 & 0.804447 & 0.8044464859485744 & $6.70233 \times 10^{-7}$ & $8.33160 \times 10^{-7}$ \\
0.48 & 0.779112 & 0.7791116154224935 & $7.50275 \times 10^{-7}$ & $9.62986 \times 10^{-7}$ \\
0.64 & 0.794216 & 0.7942151498560056 & $7.02761 \times 10^{-7}$ & $8.84848 \times 10^{-7}$ \\
0.80 & 0.852144 & 0.8521432738935479 & $5.15073 \times 10^{-7}$ & $6.04443 \times 10^{-7}$ \\
0.96 & 0.962328 & 0.9623277968729329 & $1.35849 \times 10^{-7}$ & $1.41167 \times 10^{-7}$ \\
\hline
\end{tabular}

TABLE 3: Absolute error of approximating $u_{1}^{\prime}(x)$ and $u_{2}^{\prime}(x)$ for Example 1.

\begin{tabular}{lcccc}
\hline Derivative & $x=0.16$ & $x=0.48$ & $x=0.64$ & $x=0.96$ \\
\hline$u_{1}^{\prime}(x)$ & $3.96943 \times 10^{-6}$ & $4.14991 \times 10^{-6}$ & $4.09071 \times 10^{-6}$ & $3.7627 \times 10^{-6}$ \\
$u_{2}^{\prime}(x)$ & $8.88178 \times 10^{-7}$ & $3.15362 \times 10^{-6}$ & $1.11022 \times 10^{-6}$ & $2.10942 \times 10^{-7}$ \\
\hline
\end{tabular}

TABLE 4: Numerical results of $u_{1}(x)$ for Example 2.

\begin{tabular}{lcccc}
\hline$x$ & Exact solution & Approximate solution & Absolute error & Relative error \\
\hline 0.16 & 0.0180634 & 0.01806252000000006 & $8.39999 \times 10^{-7}$ & $4.65029 \times 10^{-5}$ \\
0.32 & 0.0473498 & 0.04734840000000003 & $1.35999 \times 10^{-6}$ & $2.87224 \times 10^{-5}$ \\
0.48 & 0.0623002 & 0.06229859999999997 & $1.56000 \times 10^{-6}$ & $2.50401 \times 10^{-5}$ \\
0.64 & 0.0530842 & 0.05308272000000007 & $1.43999 \times 10^{-6}$ & $2.71267 \times 10^{-5}$ \\
0.80 & 0.0256000 & 0.02559900000000005 & $9.99999 \times 10^{-7}$ & $3.90625 \times 10^{-5}$ \\
0.96 & 0.0014746 & 0.00147432000000012 & $2.39999 \times 10^{-7}$ & $1.62760 \times 10^{-4}$ \\
\hline
\end{tabular}

TABLE 5: Numerical results of $u_{2}(x)$ for Example 2.

\begin{tabular}{lcccc}
\hline$x$ & Exact solution & Approximate solution & Absolute error & Relative error \\
\hline 0.16 & 0.982257 & 0.982258015821409 & $8.80077 \times 10^{-7}$ & $8.95974 \times 10^{-7}$ \\
0.32 & 0.954791 & 0.954792235061675 & $1.35412 \times 10^{-6}$ & $1.41824 \times 10^{-6}$ \\
0.48 & 0.941354 & 0.941355026122341 & $1.50133 \times 10^{-6}$ & $1.59487 \times 10^{-6}$ \\
0.64 & 0.949592 & 0.949593136843461 & $1.41570 \times 10^{-6}$ & $1.49085 \times 10^{-6}$ \\
0.80 & 0.975039 & 0.975040039211652 & $1.03765 \times 10^{-6}$ & $1.06422 \times 10^{-6}$ \\
0.96 & 0.998528 & 0.998527858662958 & $2.47537 \times 10^{-7}$ & $2.47902 \times 10^{-7}$ \\
\hline
\end{tabular}

TABLE 6: Numerical results of $u_{1}(x)$ for Example 3.

\begin{tabular}{lcccc}
\hline$x$ & Exact solution & Approximate solution & Absolute error & Relative error \\
\hline 0.16 & -0.144332 & -0.1443317288548306 & $6.42035 \times 10^{-7}$ & $4.44831 \times 10^{-6}$ \\
0.32 & -0.245389 & -0.2453879153282803 & $1.24493 \times 10^{-6}$ & $5.07330 \times 10^{-6}$ \\
0.48 & -0.287149 & -0.2871473420226861 & $1.53927 \times 10^{-6}$ & $5.36052 \times 10^{-6}$ \\
0.64 & -0.261884 & -0.2618830218641385 & $1.35777 \times 10^{-6}$ & $5.18460 \times 10^{-6}$ \\
0.80 & -0.174353 & -0.1743525808946390 & $8.06250 \times 10^{-7}$ & $4.62423 \times 10^{-6}$ \\
0.96 & -0.0391567 & -0.0391565628696831 & $1.52332 \times 10^{-7}$ & $3.89030 \times 10^{-6}$ \\
\hline
\end{tabular}


TABle 7: Numerical results of $u_{2}(x)$ for Example 3.

\begin{tabular}{lcccc}
\hline$x$ & Exact solution & Approximate solution & Absolute error & Relative error \\
\hline 0.16 & 1.14385 & 1.143849565677054 & $7.02579 \times 10^{-7}$ & $6.14223 \times 10^{-7}$ \\
0.32 & 1.24309 & 1.243088505342329 & $1.22692 \times 10^{-6}$ & $9.86995 \times 10^{-7}$ \\
0.48 & 1.28351 & 1.283510460180459 & $1.44905 \times 10^{-6}$ & $1.12897 \times 10^{-6}$ \\
0.64 & 1.25910 & 1.259102236286934 & $1.31435 \times 10^{-6}$ & $1.04388 \times 10^{-6}$ \\
0.80 & 1.17351 & 1.173510014918347 & $8.56073 \times 10^{-7}$ & $7.29498 \times 10^{-7}$ \\
0.96 & 1.03915 & 1.039146624444131 & $1.84037 \times 10^{-7}$ & $1.77104 \times 10^{-7}$ \\
\hline
\end{tabular}

TABLE 8: Numerical results of $u_{3}(x)$ for Example 3.

\begin{tabular}{lcccc}
\hline$x$ & Exact solution & Approximate solution & Absolute error & Relative error \\
\hline 0.16 & 0.874645 & 0.8746445398520759 & $7.43544 \times 10^{-7}$ & $8.50109 \times 10^{-7}$ \\
0.32 & 0.806168 & 0.8061672349997103 & $1.20922 \times 10^{-6}$ & $1.49996 \times 10^{-6}$ \\
0.48 & 0.781712 & 0.7817107460286419 & $1.39143 \times 10^{-6}$ & $1.77998 \times 10^{-6}$ \\
0.64 & 0.796259 & 0.7962584197762563 & $1.28185 \times 10^{-6}$ & $1.60984 \times 10^{-6}$ \\
0.80 & 0.852827 & 0.8528264441003238 & $8.85879 \times 10^{-7}$ & $1.03876 \times 10^{-6}$ \\
0.96 & 0.962337 & 0.9623371578286029 & $2.12773 \times 10^{-7}$ & $2.21100 \times 10^{-7}$ \\
\hline
\end{tabular}

$$
\begin{aligned}
f_{1}(x)=(\cosh (x(x-1))+x(x-1))(x(x-1)+1) \\
+e^{-2 x(x-1)}+\frac{2 x-1}{x(x-1)+1} \\
\begin{aligned}
f_{2}(x)= & (\cosh (x(x-1))+x(x-1))^{2} \\
& -e^{-x(x-1)}(2 x-1)-\frac{e^{-x(x-1)}}{x(x-1)+1}, \\
& =\sinh (x(x-1))(2 x-1) \\
f_{3}(x)= & (\cosh (x(x-1))+x(x-1)) \\
& \times \ln (x(x-1)+1) e^{-x(x-1)}+2 x-1
\end{aligned}
\end{aligned}
$$

subject to the periodic boundary conditions

$$
\begin{aligned}
& u_{1}(0)=u_{1}(1), \\
& u_{2}(0)=u_{2}(1), \\
& u_{3}(0)=u_{3}(1) .
\end{aligned}
$$

The exact solutions are $u_{1}(x)=\ln (x(x-1)+1), u_{2}(x)=$ $e^{x(1-x)}$, and $u_{3}(x)=x(x-1)+\cosh (x(x-1))$.

Using RKHS method, take $x_{i}=(i-1) /(M-1), i=$ $1,2, \ldots, M$, on $[0,1]$. The numerical results at some selected grid points for $M=101$ and $m=3$ are given in Tables 6 , 7 , and 8 for the dependent variables $u_{1}(x), u_{2}(x)$, and $u_{3}(x)$, respectively.

From the previous tables, it can be seen that the RKHS method provides us with the accurate approximate solutions. On the other aspect as well, it is clear that the accuracy obtained using the mentioned method is advanced by using only a few tens of iterations.

\section{Conclusions}

Here, we use the RKHS method to solve systems of firstorder periodic BVPs. The solutions were calculated in the form of a convergent series in the space $W_{2}^{2}[0,1]$ with easily computable components. In the proposed method, the $m$-term approximations are obtained and proved to converge to the exact solutions. Meanwhile, the error of the approximate solutions is monotone decreasing in the sense of the norm of $W_{2}^{2}[0,1]$. It is worthy to note that, in our work, the approximate solutions and their derivatives converge uniformly to the exact solutions and their derivatives, respectively. On the other aspect as well, the present method enables us to approximate the solutions and their derivatives at every point of the range of integration. The results show that the present method is an accurate and reliable analytical technique for solving systems of first-order periodic BVPs.

\section{Conflict of Interests}

The authors declare that there is no conflict of interests.

\section{Acknowledgment}

The authors would like to express their thanks to unknown referees for their careful reading and helpful comments.

\section{References}

[1] E. Coddington and N. Levinson, Theory of Ordinary Differential Equations, McGraw-Hill, New York, NY, USA, 1955.

[2] H. I. Freedman and J. H. Wu, "Periodic solutions of singlespecies models with periodic delay," SIAM Journal on Mathematical Analysis, vol. 23, no. 3, pp. 689-701, 1992.

[3] J. Mawhin and J. R. Ward, "Nonuniform nonresonance conditions at the two first eigenvalues for periodic solutions of 
forced Lienard and Duffing equations," Rocky Mountain Journal of Mathematics, vol. 12, no. 4, pp. 643-654, 1982.

[4] A. C. Lazer, "Application of a lemma on bilinear forms to a problem in nonlinear oscillations," Proceedings of the American Mathematical Society, vol. 33, pp. 89-94, 1972.

[5] K. Abd-Ellateef, R. Ahmed, and Z. Drici, "Generalized quasilinearization for systems of nonlinear differential equations with periodic boundary conditions," Dynamics of Continuous, Discrete \& Impulsive Systems A, vol. 12, no. 1, pp. 77-85, 2005.

[6] C. C. Tisdell, "Existence of solutions to first-order periodic boundary value problems," Journal of Mathematical Analysis and Applications, vol. 323, no. 2, pp. 1325-1332, 2006.

[7] R. P. Agarwal and J. Chen, "Periodic solutions for first order differential systems," Applied Mathematics Letters, vol. 23, no. 3, pp. 337-341, 2010.

[8] R. Chen, R. Ma, and Z. He, "Positive periodic solutions of firstorder singular systems," Applied Mathematics and Computation, vol. 218, no. 23, pp. 11421-11428, 2012.

[9] C. P. Gupta, "Periodic solutions for coupled first order nonlinear differential systems of Hamiltonian type," Nonlinear Analysis: Theory, Methods \& Applications, vol. 8, no. 11, pp. 1271-1285, 1984.

[10] Q. Kong and M. Wang, "Positive solutions of even order system periodic boundary value problems," Nonlinear Analysis: Theory, Methods \& Applications, vol. 72, no. 3-4, pp. 1778-1791, 2010.

[11] A. Boucherif and N. Merabet, "Boundary value problems for first order multivalued differential systems," Archivum Mathematicum, vol. 41, no. 2, pp. 187-195, 2005.

[12] J. M. Ortega and W. C. Rheinboldt, Iterative Solution of Nonlinear Equations in Several Variables, Academic Press, New York, NY, USA, 1970.

[13] W. G. Li, "Solving the periodic boundary value problem with the initial value problem method," Journal of Mathematical Analysis and Applications, vol. 226, no. 1, pp. 259-270, 1998.

[14] J. Mawhin, Topological Degree Methods in Nonlinear Boundary Value Problems, vol. 40 of CBMS Regional Conference Series in Mathematics, American Mathematical Society, Providence, RI, USA, 1979.

[15] I. T. Kiguradze, "On periodic solutions of $n$th order ordinary differential equations," Nonlinear Analysis: Theory, Methods \& Applications, vol. 40, no. 1-8, pp. 309-321, 2000.

[16] O. abu Arqub, A. el-Ajou, Z. al Zhour, and S. Momani, "Multiple solutions of nonlinear boundary value problems of fractional order: a new analytic iterative technique," Entropy, vol. 16, no. 1, pp. 471-493, 2014.

[17] Z. abo-Hammour, O. abu Arqub, S. Momani, and N. Shawagfeh, "Optimization solution of Troesch's and Bratu's problems of ordinary type using novel continuous genetic algorithm," Discrete Dynamics in Nature and Society, vol. 2014, Article ID 401696, 15 pages, 2014.

[18] O. abu Arqub, Z. abo-Hammour, and S. Momani, "Application of continuous genetic algorithm for nonlinear system of second-order boundary value problems," Applied Mathematics and Information Sciences, vol. 8, pp. 235-248, 2014.

[19] O. abu Arqub, Z. abo-Hammour, S. Momani, and N. Shawagfeh, "Solving singular two-point boundary value problems using continuous genetic algorithm," Abstract and Applied Analysis, vol. 2012, Article ID 205391, 25 pages, 2012.

[20] A. Berlinet and C. Thomas-Agnan, Reproducing Kernel Hilbert Spaces in Probability and Statistics, Kluwer Academic, Boston, Mass, USA, 2004.
[21] M. Cui and Y. Lin, Nonlinear Numerical Analysis in the Reproducing Kernel Space, Nova Science, New York, NY, USA, 2008.

[22] A. Daniel, Reproducing Kernel Spaces and Applications, Springer, Basel, Switzerland, 2003.

[23] F. Geng, "Solving singular second order three-point boundary value problems using reproducing kernel Hilbert space method," Applied Mathematics and Computation, vol. 215, no. 6, pp. 2095-2102, 2009.

[24] L.-H. Yang and Y. Lin, "Reproducing kernel methods for solving linear initial-boundary-value problems," Electronic Journal of Differential Equations, vol. 2008, pp. 1-11, 2008.

[25] Y.Z. Lin, M. G. Cui, and L. H. Yang, "Representation of the exact solution for a kind of nonlinear partial differential equation," Applied Mathematics Letters, vol. 19, no. 8, pp. 808-813, 2006.

[26] W. Wang, M. Cui, and B. Han, "A new method for solving a class of singular two-point boundary value problems," Applied Mathematics and Computation, vol. 206, no. 2, pp. 721-727, 2008.

[27] W. Jiang and Z. Chen, "Solving a system of linear Volterra integral equations using the new reproducing kernel method," Applied Mathematics and Computation, vol. 219, no. 20, pp. 10225-10230, 2013.

[28] F. Geng and M. Cui, "A reproducing kernel method for solving nonlocal fractional boundary value problems," Applied Mathematics Letters, vol. 25, no. 5, pp. 818-823, 2012.

[29] F. Z. Geng and S. P. Qian, "Reproducing kernel method for singularly perturbed turning point problems having twin boundary layers," Applied Mathematics Letters, vol. 26, no. 10, pp. 998-1004, 2013.

[30] W. Jiang and Z. Chen, "A collocation method based on reproducing kernel for a modified anomalous subdiffusion equation," Numerical Methods for Partial Differential Equations, vol. 30, no. 1, pp. 289-300, 2014.

[31] F. Z. Geng, S. P. Qian, and S. Li, "A numerical method for singularly perturbed turning point problems with an interior layer," Journal of Computational and Applied Mathematics, vol. 255, pp. 97-105, 2014.

[32] N. Shawagfeh, O. abu Arqub, and S. Momani, "Analytical solution of nonlinear second-order periodic boundary value problem using reproducing kernel method," Journal of Computational Analysis and Applications, vol. 16, pp. 750-762, 2014.

[33] M. al-Smadi, O. abu Arqub, and S. Momani, "A computational method for two-point boundary value problems of fourth-order mixed integrodifferential equations," Mathematical Problems in Engineering, vol. 2013, Article ID 832074, 10 pages, 2013.

[34] O. abu Arqub, M. al-Smadi, and N. Shawagfeh, "Solving Fredholm integro-differential equations using reproducing kernel Hilbert space method," Applied Mathematics and Computation, vol. 219, no. 17, pp. 8938-8948, 2013.

[35] O. abu Arqub, M. al-Smadi, and S. Momani, "Application of reproducing kernel method for solving nonlinear FredholmVolterra integrodifferential equations," Abstract and Applied Analysis, vol. 2012, Article ID 839836, 16 pages, 2012. 


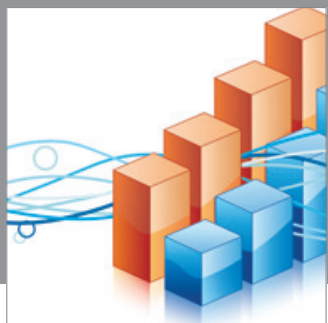

Advances in

Operations Research

mansans

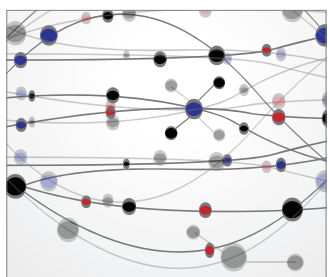

The Scientific World Journal
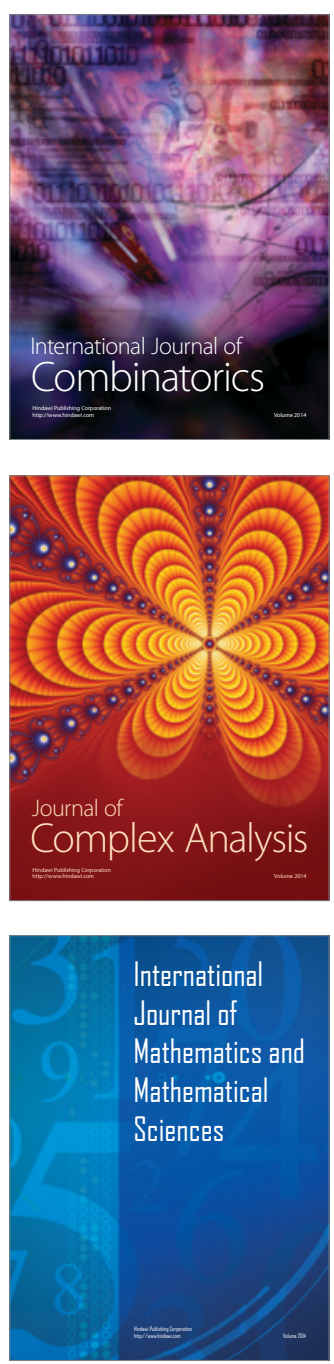
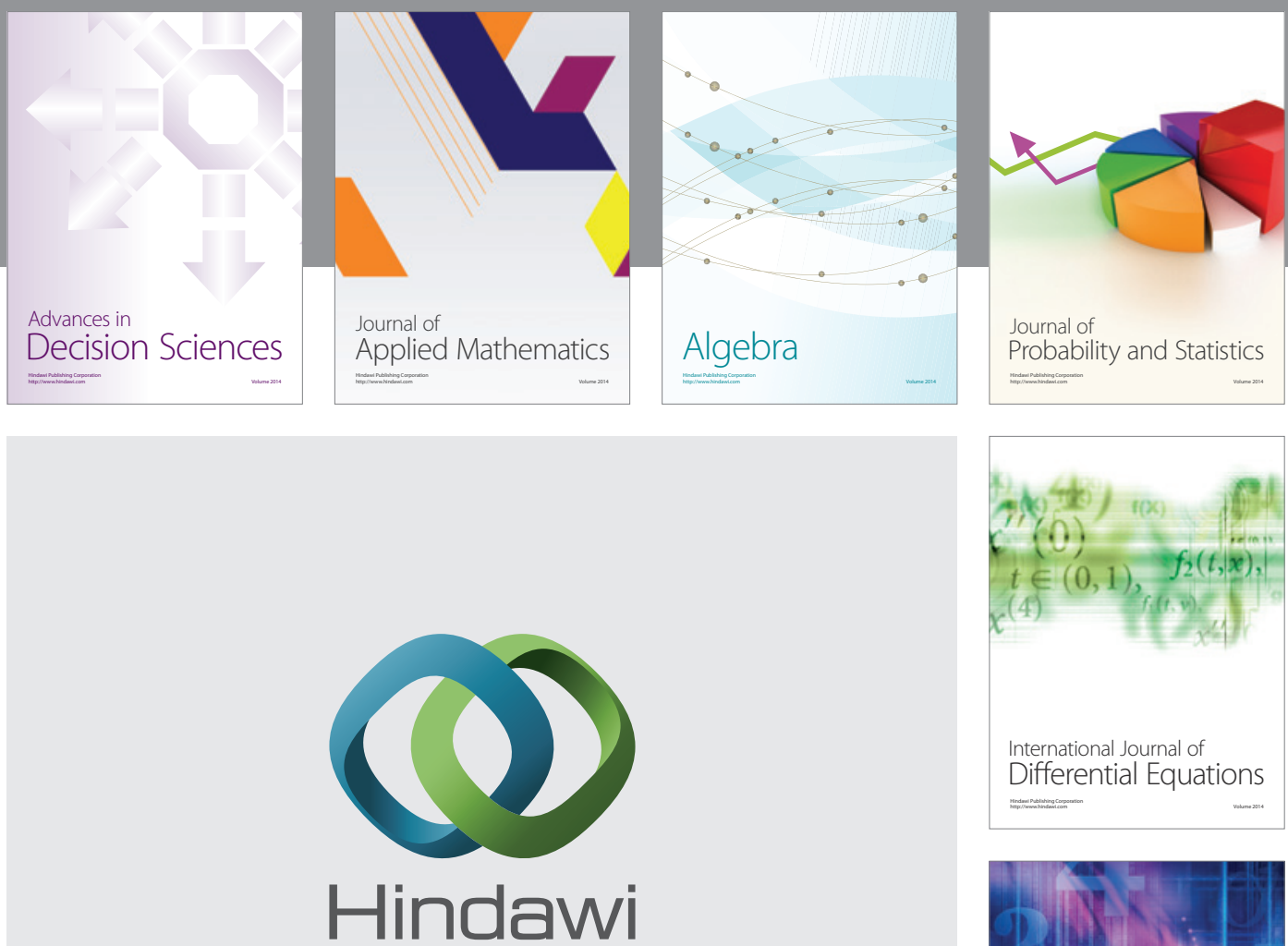

Submit your manuscripts at http://www.hindawi.com
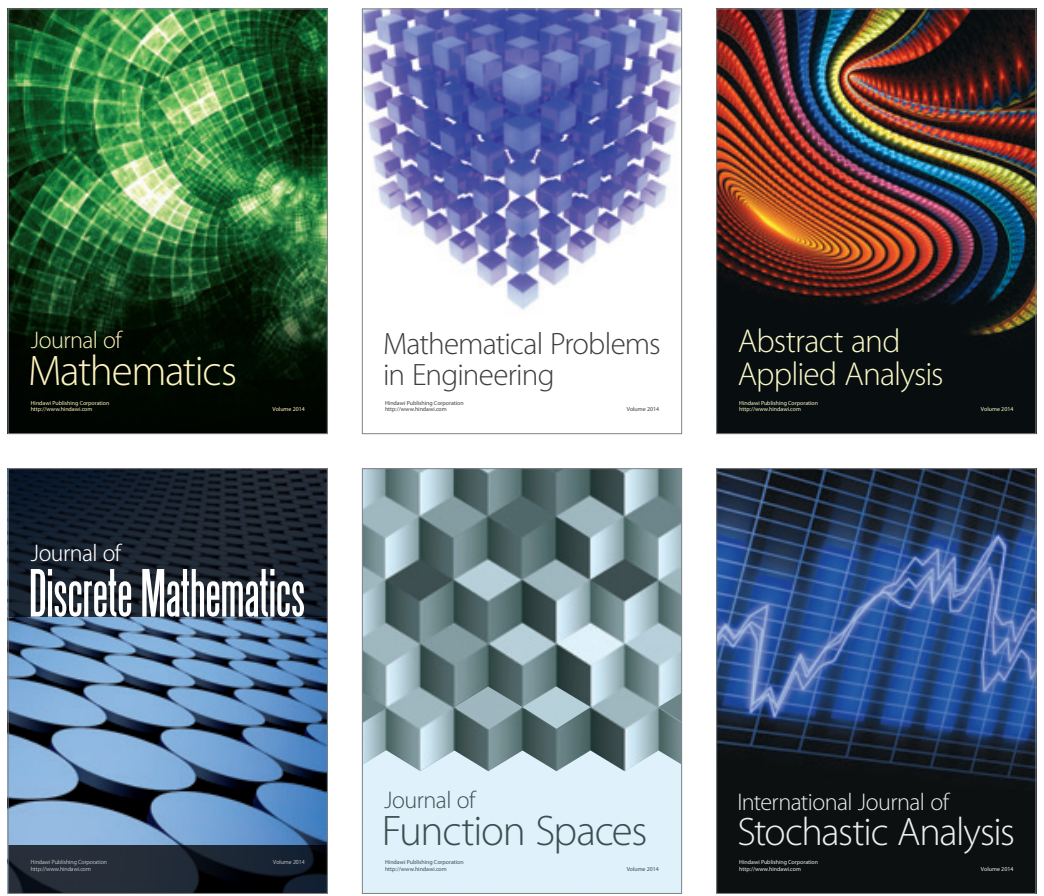

Journal of

Function Spaces

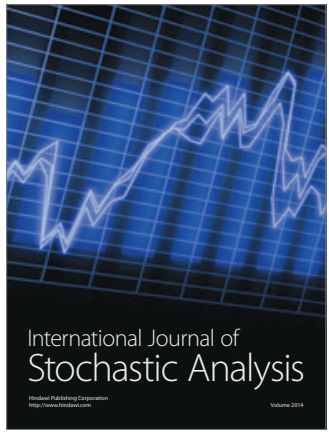

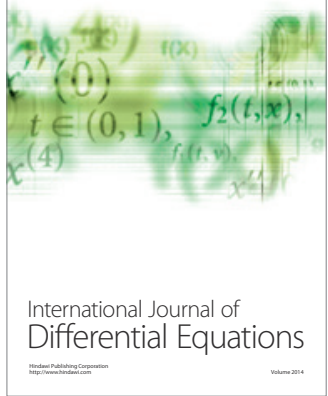
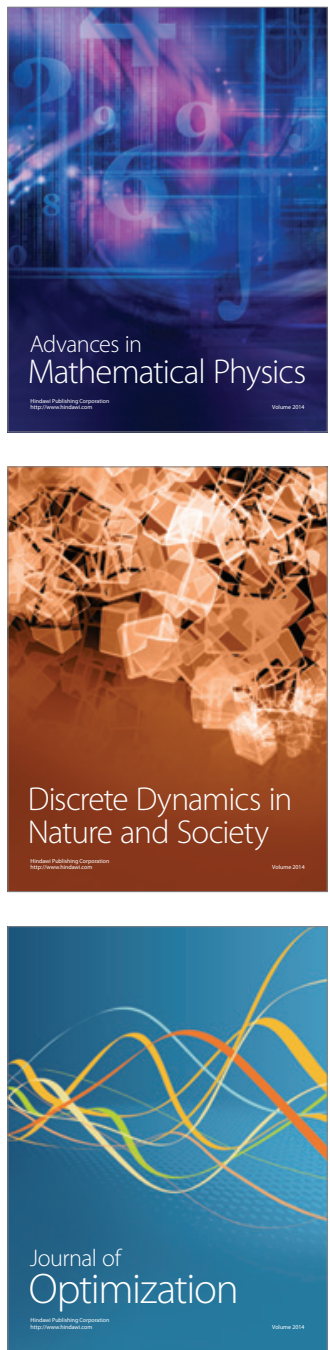